\title{
Germinated barley foodstuff suppresses dextran sulfate experimental colitis in rats: The role of mast cells
}

\author{
YOSHIO ARAKI $^{1}$, OSAMU KANAUCHI ${ }^{2}$, HIROYUKI SUGIHARA ${ }^{1}$, \\ YOSHIHIDE FUJIYAMA ${ }^{3}$ and TAKANORI HATTORI ${ }^{1}$ \\ ${ }^{1}$ First Department of Pathology, Shiga University of Medical Science, Seta Tsukinowa, Otsu, Shiga 520-2192; \\ ${ }^{2}$ Research and Development Planning Department of the Functional Food Division, Kirin Brewery Co., Ltd., \\ 10-1-2 Shinkawa Chuo-ku, Tokyo 104-8288; ${ }^{3}$ Department of Internal Medicine, Shiga University \\ of Medical Science, Seta Tsukinowa, Otsu, Shiga 520-2192, Japan
}

Received July 31, 2006; Accepted September 29, 2006

\begin{abstract}
Recent studies have suggested that dietary fiber exerts a therapeutic effect on inflammatory bowel disease patients. The aim of this study was to evaluate the effects of a dietary fiber, germinated barley foodstuff $(\mathrm{GBF})$, derived from the aleurone and scutellum fraction of germinated barley against dextran sulfate sodium (DSS)-induced experimental colitis in rats. Sprague-Dawley rats were fed a $3 \%$ DSS diet containing GBF or $\alpha$-cellulose for 8 days. The mucosal damage (macroscopic and microscopic inflammation) was then quantified. In addition, we evaluated the alterations in the mucosal mast cells and connective tissues. GBF effectively prevented mucosal damage. In addition, GBF suppressed the infiltration of the mucosal mast cells, and prevented the distraction of both collagen and elastic fibers. These effects may be closely associated with its inhibitory effects on mucosal mast cells, and the destruction of the mucosal connective tissues.
\end{abstract}

\section{Introduction}

The pathogenesis of inflammatory bowel disease (IBD), ulcerative colitis (UC) and Crohn's disease (CD) remain unknown. At present, corticosteroids and sulfasalazine are commonly used for the treatment of IBD patients (1). However, the use of these agents is limited by their adverse effects (1). On the other hand, there is a type of dietary therapy often used in enteral feeding for the treatment of active CD patients, but its effects are controversial (2).

Short chain fatty acids (SCFAs) such as acetic, propionic and butyric acids, are known to be produced essentially by

Correspondence to: Dr Yoshio Araki, 6-13-20 Aoyama, Otsu, Shiga 520-2101, Japan

E-mail: bileacidaraki@hotmail.com

Key words: germinated barley foodstuff, dextran sulfate sodiuminduced colitis, mast cells, connective tissues the anaerobic fermentation of carbohydrates, including dietary fiber, resistant starch and mucus, in the colon. SCFAs exert a variety of physiologic effects (3). Some studies have reported the clinical effects of dietary fiber and/or SCFAs as therapeutic tools for IBD patients $(3,4)$. For example, SCFA enemas were performed in patients with left-sided UC, and their efficacy was demonstrated. However, the major limitation is that the enema flow only reaches as far as the splenic flexure (5), and its retention time in the colon is short (3). We have previously reported that a certain kind of dietary fiber, germinated barley foodstuff (GBF), is the best generator of SCFAs in the gut lumen, and exerts strong therapeutic effects against experimental colitis in rats (6) and clinical UC patients $(7,8)$. With respect to the mechanism of action of GBF, we discovered that GBF has glutamine-rich proteins, has a high binding capacity for cytotoxic bile acids (9), and a high capacity for SCFA production through the actions of Eubacterium and Bifidobacterium in the gut lumen, and that the levels of SCFA correlated to an amelioration of the colitis (6), and inactivated nuclear factor kappa B (NFкB) (10).

The aim of the present study was to evaluate the effects of GBF against dextran sulfate sodium (DSS; sulfated polysaccharide)-induced experimental colitis in rats, an animal model which exhibits colonic lesions similar to human UC (11). In particular, we focused on the role of mast cells and the alterations in the connective tissues in the intestinal mucosa.

\section{Materials and methods}

Germinated barley foodstuff. The process of producing GBF (KRD-7181, Kirin Brewery Co., Gunma, Japan) is described below. After the germination process, the barley is mashedfiltered to extract the endosperm as a substrate for fermentation by yeast. Then the residual insoluble fraction, the brewer's spent grain, is obtained. GBF represents the aleurone and scutellum fraction obtained by milling and sieving the residual brewer's spent grain $(12,13)$. GBF contains both glutamine-rich proteins and dietary fibers, mainly hemicellulose. The chemical composition of GBF is shown in 

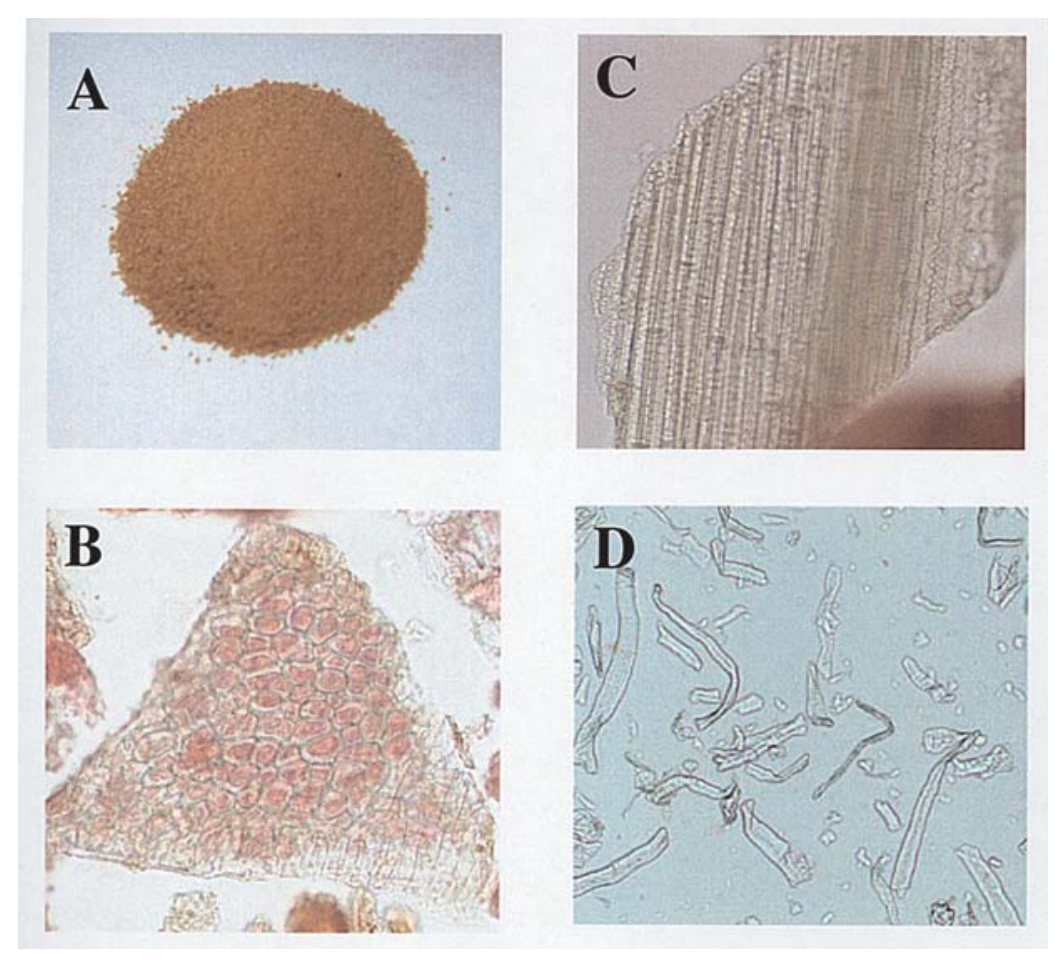

Figure 1. Macroscopic and microscopic features of GBF. After $80 \mathrm{mg}$ of germinated barley foodstuff (GBF) or $\alpha$-cellulose fiber was immersed in distilled water for $30 \mathrm{~min}$, the sediment was added to a melted $5 \%$ agar solution. After mechanical shaking, the mixture was cooled in ice water. Blocks of the solid mixture were then frozen under liquid nitrogen, and sliced on a cryostat. The samples were then stained with hematoxylin and eosin (H\&E). (A) Macroscopic features of GBF; (B) and (C) microscopic features of GBF; and (D) macroscopic features of $\alpha$-cellulose. Magnification x200.

Table I. Chemical composition of germinated barley foodstuff.

\begin{tabular}{lc}
\hline & $\%$ Weight \\
\hline Water & 7.8 \\
Protein & 46.0 \\
Lipids & 10.2 \\
Ash & 2.0 \\
Dietary fiber & 34.0 \\
Nitrogen-free extracts & b \\
\hline
\end{tabular}

${ }^{a}$ As neutral detergent fiber. ${ }^{\text {b}}$ This fraction mainly contains watersoluble mono- or oligo-saccharides. This value was calculated as follows: 100 -(water content+lipid content+dietary fiber content+ash content) $(\%)$.

Table I. GBF contains about $30 \%$ insoluble dietary fiber by weight. Fig. 1A shows the macroscopic features of GBF. Fig. 1B, C and D show the microscopic features of GBF and the cellulose fibers. GBF consists mainly of aleurone, which has a lattice-like structure and encloses cytoplasmic elements in the center (Fig. 1B). In addition, a small amount of husk, which has a plate-like structure, is present (Fig. 1C). On the other hand, $\alpha$-cellulose mainly shows a fibrillar form of fiber (Fig. 1D) (9). To note, GBF neither binds to DSS nor eliminates DSS in the gut lumen (6).

Animals. Specific pathogen-free male Sprague-Dawley rats, 11 weeks old (400-450 g), were purchased from Nippon Clea Inc. (Tokyo, Japan). They were housed in a room with controlled temperature $\left(20-22^{\circ} \mathrm{C}\right)$, humidity (50-60\%) and a preset light-dark cycle $(12 \mathrm{~h} / 12 \mathrm{~h})$. The experimental protocol was approved by the Animal Care and Use Committee of the Shiga University of Medical Science.

Rat DSS colitis. The rats were allowed food and drinking water ad libitum, and were initially divided into three groups of 6 rats. The rats were fed $\alpha$-cellulose (CE+DSS) and GBF (GBF+DSS) containing 3\% (w/w of diet) DSS (molecular weight 5000, total sulfur 15.0-20.0\%; Wako Pure Chemical, Osaka, Japan) for 8 days. The control rats were fed cellulose alone for 8 days. The composition of the respective diets is shown in Table II. During the experimental period, food intake and body weight were measured daily.

Macroscopic mucosal damaged area. On the final day of the experiment, the rats were anesthetized with an intraperitoneal injection of pentobarbital sodium $(40 \mathrm{mg} / \mathrm{kg})$. After the rats were sacrificed by cervical dislocation, the body weight was measured and a laparotomy was performed. The large intestine from the anus to the cecocolonic junction was resected, irrigated with chilled saline and then cut along the antemesenteric border. The mucosa was photographed and the area of damage (color change to purple and red, structural changes with irregular folds, edema and erosion) was determined using an image analysis apparatus (NIH image version 4.0/Macintosh) for macroscopic evaluation according to our previous report (14).

Damage score. A tissue specimen $(10 \times 10 \mathrm{~mm})$ was removed $4 \mathrm{~cm}$ (middle portion) from the anal margin, fixed in Carnoy's fixative, and embedded in paraffin. Histological samples 


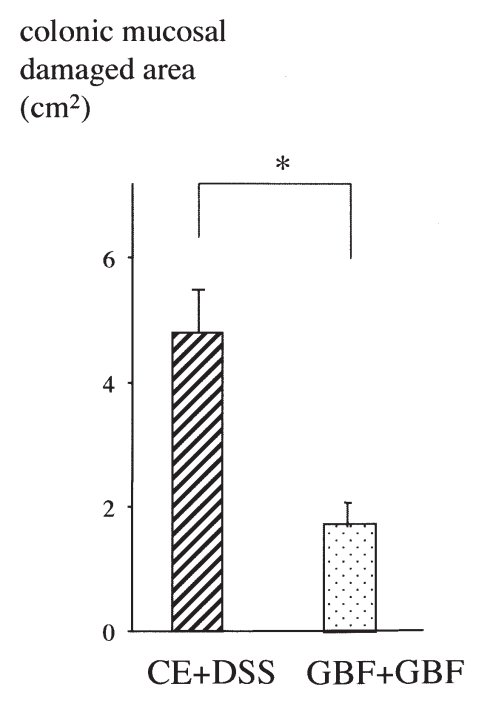

A damage score

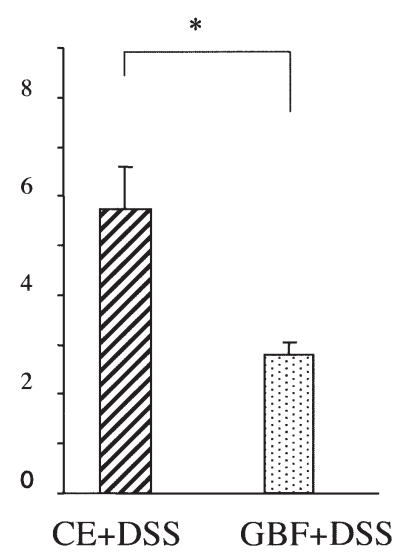

B

Figure 2. Mucosal damaged area and damage scores of the colon. After the rats were fed experimental diets for 8 days, the colon was photographed and the area of damage was determined using an image analysis apparatus. (A) The damaged areas in the CE+DSS and GBF+DSS groups are shown. A tissue specimen was also removed $1 \mathrm{~cm}$ from the anal margin, and fixed in Carnoy's fixative. The histological samples were then stained with hematoxylin and eosin (H\&E). The mucosal damage score was determined according to a previously described method (16). (B) Damage scores from the CE+DSS and GBF+DSS groups are shown. The values are expressed as means $\pm \mathrm{SEM},{ }^{*} \mathrm{p}<0.05$.

Table II. Composition of experimental diets ( $\mathrm{g} / \mathrm{kg}$ diet).

\begin{tabular}{lcc}
\hline & CE & GBF \\
\hline Casein & 146.0 & 100.0 \\
Vitamin mixture $^{\mathrm{a}}$ & 10.0 & 10.0 \\
Mineral mixture $^{\mathrm{a}}$ & 35.0 & 35.0 \\
Choline chloride $_{\text {Cellulose }}$ & 2.0 & 2.0 \\
GBF & 30.0 & - \\
DSS & - & 100.0 \\
Corn oil & 30.0 & 30.0 \\
Corn starch $_{\text {Others }}^{\mathrm{b}}$ & 50.0 & 50.0 \\
\hline
\end{tabular}

Basic diets containing cellulose (CE) and germinated barley foodstuff (GBF). DSS, dextran sulfate sodium. ${ }^{a}$ Vitamin and mineral mixtures were prepared according to the American Institute

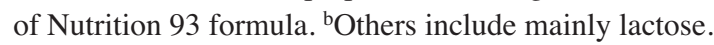

were then cut into 5- $\mu \mathrm{m}$ sections, and stained with hematoxylin and eosin (H\&E) after de-paraffinization. The mucosal damage was evaluated independently by two investigators blinded to the study groups, and was determined according to a previously described method (15). The scores derived from the two investigators were averaged. The following three parameters were used to quantify the damage; surface epithelial loss, crypt destruction and inflammatory cell infiltration into the mucosa. A score of 0-4 was assigned to each of the three parameters according to the extent and severity of the changes as follows: $0=$ no change; $1=$ localized and mild; $2=$ localized and moderate; $3=$ extensive and moderate; and $4=$ extensive and severe. The sum of the scores from the three parameters was defined as the mucosal damage score for each animal.

Observation of mast cells and connective tissues in the intestinal mucosa. Toluidine blue staining ( $\mathrm{pH} \mathrm{2.5)} \mathrm{was}$ performed to evaluate the mast cells in the intestine (14). Total mast cell counts in the intestinal wall were also numerated from the anus to $1 \mathrm{~cm}$ from the oral side. In addition, we evaluated the relationship between the local mast cell counts and the local mucosal damage in the intestines of both the CE+DSS and GBF+DSS groups. Briefly, we randomly selected intestinal fields under x100 magnification, and counted the number of local mast cell in these fields. We then evaluated the local mucosal damage according to the following three parameters; surface epithelial loss, crypt destruction, and inflammatory cell infiltration into the mucosa. A score of 0-3 was assigned to each of the three parameters according to the severity of the change as follows: $0=$ no change; $1=$ mild; $2=$ moderate; and $3=$ severe. The sum of the scores from the three parameters was defined as the local mucosal damage for each field.

In addition, elastica van Gieson and silver impregnation stains were performed to investigate the collagen and elastic fibers, and the reticulin fibers, respectively.

Statistics. The results are presented as means \pm SEM. Differences between means were evaluated using the MannWhitney $U$ test for analysis. Differences were regarded as statistically significant for $\mathrm{p}$ values $<0.05$.

\section{Results}

Macroscopic mucosal damaged area. The dietary intakes of all groups were generally comparable $(31.5 \pm 7.2 \mathrm{~g} /$ day, 

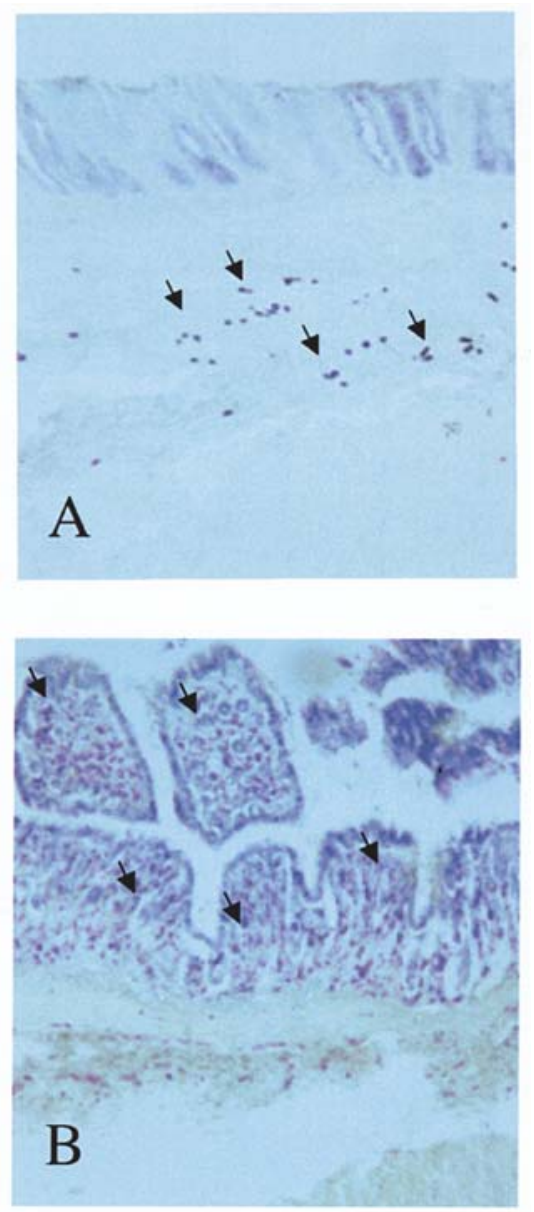

Figure 3. Light microscopic photographs using toluidine blue staining. The histological samples were stained with toluidine blue $(\mathrm{pH} 2.5)$ to detect mast cells or macrophages. (A) Mast cells (arrows) were detected in the colonic walls in both rats. (B) Many macrophages (arrows) containing ingested DSS particles exhibiting metachromasia were detected in the colonic walls in both rats. Magnification x200.

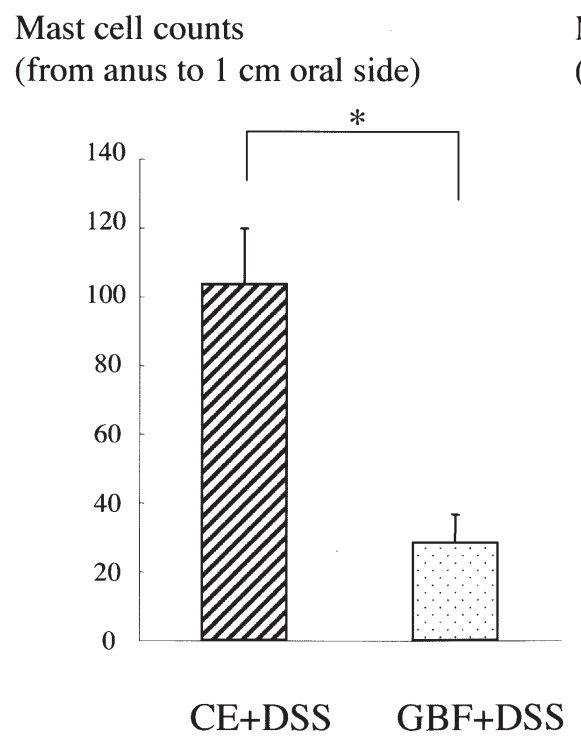

A
$28.6 \pm 5.2 \mathrm{~g} / \mathrm{day}$ and $29.4 \pm 7.8 \mathrm{~g} /$ day in the control group, CE+DSS group, and GBF+DSS group, respectively). No significant differences in body weight gain between the groups were observed during the experimental period. In the CE+DSS group, diarrhea occurred on days 2-3, and macroscopic bloody stools appeared on days 5-6. The GBF+DSS group, however, showed minimal symptoms compared to the CE+DSS group. In the GBF+DSS group, diarrhea occurred on days 5-6 and bloody stools appeared on days 7-8. None of the rats in any of the treated groups died during the experimental period.

Macroscopic examination of the colon revealed hyperemia, erosion and occasional tiny blood clots in the rectum of both the CE+DSS and GBF+DSS groups. However, this mucosal damaged area was significantly reduced in the GBF+DSS group compared to the CE+DSS group (Fig. 2A).

Damage scores. Using H\&E staining, there was obvious evidence of inflammatory cell infiltration into the mucosa and submucosa in the distal portions of the CE+DSS and GBF+DSS groups. Entire crypt loss, surface epithelial loss and mucosal edema were also evident. However, these inflammatory parameters in the GBF+DSS group were significantly attenuated as compared to the CE+DSS group. The total damage scores quantitated by the scoring system are shown in Fig. 2B. The total damage scores in the GBF+DSS group were significantly lower than those in the $\mathrm{CE}+\mathrm{DSS}$ group.

Observation of mast cells. Toluidine blue staining revealed that some mast cells and numerous macrophages ingesting DSS particles with metachromasia were scattered throughout the mucosa and submucosa of the CE+DSS and GBF+DSS groups (Fig. 3). Mast cell counts in the control rats were recognized about $5 \mathrm{~cm}$ from the anus to $1 \mathrm{~cm}$ from the oral
Mast cell counts
(/field)

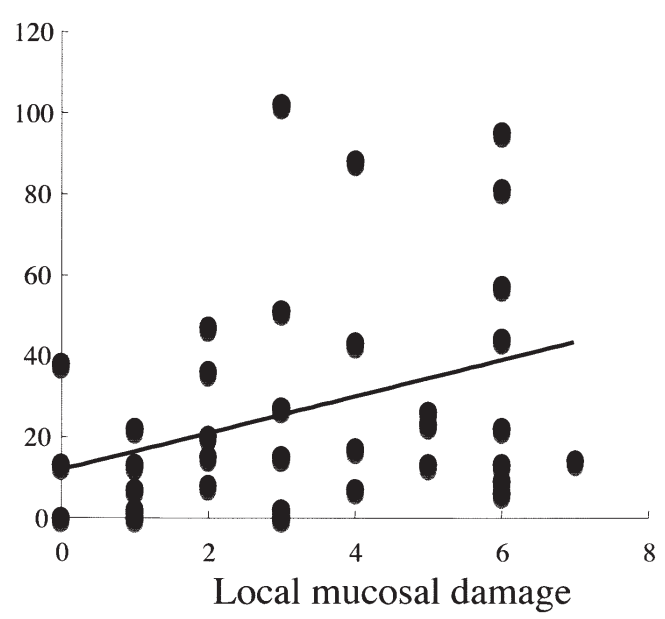

B

Figure 4. Mast cell counts and relationship to local mucosal damage. (A) Mast cell counts in the colonic wall from the anus to $1 \mathrm{~cm}$ from the oral side. The values are expressed as means \pm SEM, ${ }^{*}$ p $<0.05$. (B) Correlation between the mast cell counts and local mucosal damage. The local mucosal damage was determined according to our method described in Materials and methods. The line represents the regression line for the data from both CE+DSS- and CE+DSS-group rats $(\mathrm{y}=4.498 \mathrm{x}+11.2, \mathrm{r}=0.354, \mathrm{p}<0.05)$. 
HE

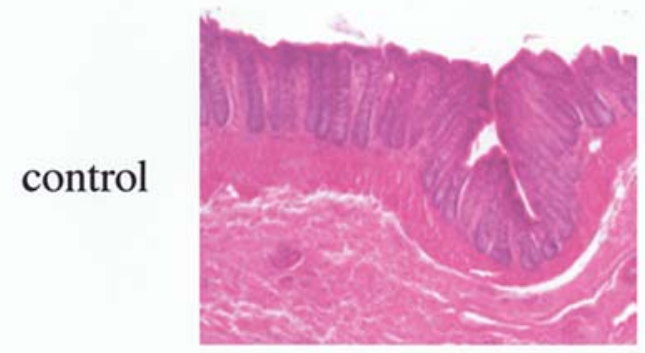

CE+DSS

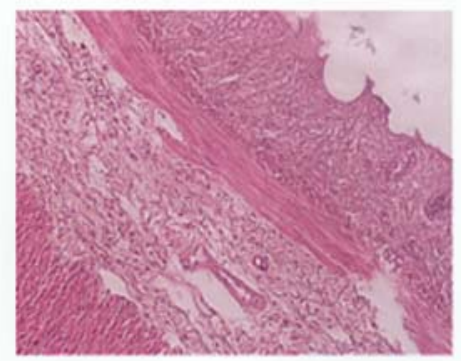

\section{GBF+DSS}

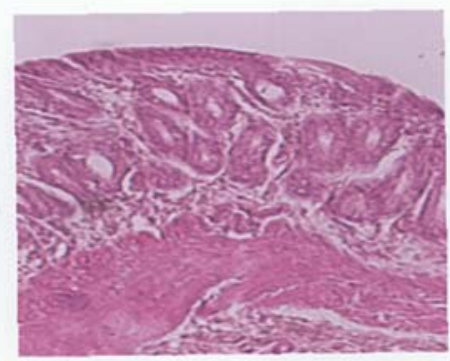

Elastica van Gieson
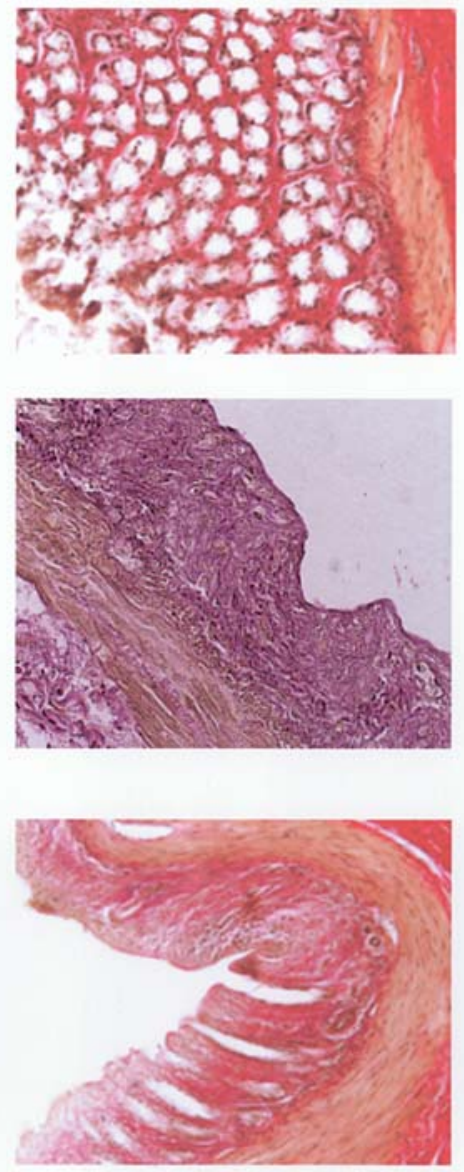

silver impregnation
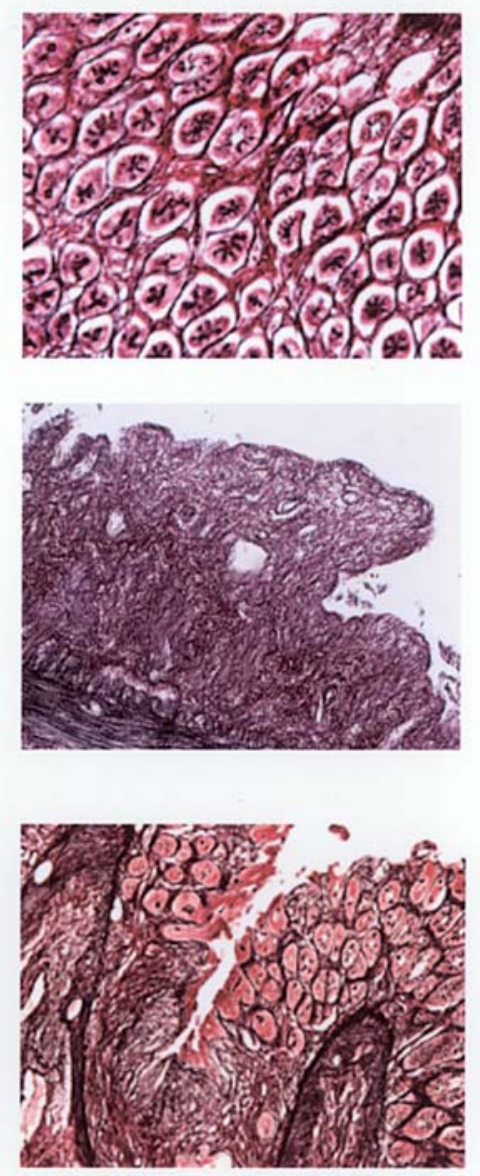

Figure 5. Light microscopic photographs using H\&E, elastica van Gieson and silver impregnation stains. The histological samples were stained with H\&E, elastica van Gieson and silver impregnation stains to investigate the collagen and elastic fibers, and the reticulin fibers, respectively. Magnifications: H\&E stain x100, elastica van Gieson and silver impregnation stains x200.

side. DSS treatment strongly increased the mast cell counts as reported in our previous study (14). The mast cell counts in the GBF+DSS group, however, were significantly lower compared to the CE+DSS group (Fig. 4A). The Pearson's correlation coefficient between the local mucosal damage and the local mast cell count was also evaluated. The local mucosal damage score had a strong positive correlation with the local mast cell counts (Fig. 4B).

Connective tissue in the intestinal mucosa. Using elastica van Gieson stain, in which the collagen and elastic fibers are stained red and black, respectively (Fig. 5), DSS administration reduced the amount of red color, disrupted the bands of collagen fibers and made them more amorphous in the $\mathrm{CE}+\mathrm{DSS}$ rats. These changes may represent the degradation of collagen fibers. However, these changes were significantly reduced in the GBF+DSS group. Using this staining method, the disappearance of the crypt structures was clear.

Using the silver impregnation stains, in which the reticulin fibers are stained black, DSS administration disrupted the bands of the reticulin fibers and made them more amorphous in the $\mathrm{CE}+\mathrm{DSS}$ rats. Once again, these changes were significantly reduced in the GBF+DSS group. Using this staining method, the disappearance of the crypt structures was also clear.

\section{Discussion}

In the present study, GBF strongly suppressed DSS-induced colitis, both macroscopically (color changes, structural changes, edema and erosion) and microscopically (surface epithelial loss, crypt destruction and inflammatory cell infiltration). In terms of the connective tissue structure, GBF attenuated the DSS-induced destruction of the connective tissues. This change in the connective tissue structure is believed to be mainly due to the inflammation of the mucosa and submucosa, accompanied by strong inflammatory cell infiltration. In the future, this parameter of the connective tissue structure will be useful for evaluating intestinal damage.

On the other hand, DSS treatment strongly increased the mast cell counts compared to the non-DSS-treated rats. GBF, however, suppressed this mast cell recruitment. In addition, we demonstrated that there was a strong positive correlation between the local mast cell counts and local mucosal damage. These results strongly suggest that intestinal mast cells play important roles in the development of DSS-induced colitis, 
and that it is possible that GBF exerts its therapeutic actions via anti-inflammatory effects including the suppression of mast cells. Our results correspond to previous reports. We previously reported that intestinal mast cells play an important role in the development of DSS-colitis using $W s / W s$ rats, which have a small deletion of the $c$-kit gene, and are deficient in mast cells (14). In that study, we observed that DSS induced a local recruitment of mast cells in the colonic mucosa of $W s / W s$ rats, and the mucosal damage gradually increased in proportion to this mast cell recruitment. Previous studies also reported that DSS-induced colitis was ameliorated in the histamine-deficient, histidine decarboxylase knockout mice (16) or in rats treated with histamine H2-receptor antagonist (17).

Why did GBF suppress the recruitment of mast cells? In our previous studies, we proposed that the most probable mechanism responsible for the amelioration of DSS-induced colitis is the generation of SCFAs derived from $\operatorname{GBF}(6,18)$. GBF is rich in low-lignified hemicellulose and lignin (19), as shown in Table I, and low-lignified hemicellulose is efficiently fermented by the microflora in the gut lumen to produce SCFAs (20). In addition, GBF produced SCFAs through the actions of Eubacterium and Bifidobacterium in the gut lumen, and that the levels of SCFAs correlated to an amelioration of colitis $(6,18)$. Therefore, of the many kinds of dietary fiber, GBF has the highest potential to produce SCFAs via fermentation.

Generally speaking, SCFAs exert a variety of physiologic effects, which include serving as an energy source for colonocytes (21) and the stimulation of colonocyte proliferation (22). Butyrate is also suggested to have anti-inflammatory effects (23).

Do SCFAs affect the mast cell activation or recruitment? Few previous studies have discussed the relationship between SCFAs and mast cells $(24,25)$. Thus the precise relationship remains unclear.

In conclusion, GBF administration suppressed DSSinduced colitis in rats. It is possible that intestinal mast cells play an important role in the mechanism of action of GBF. However, whether GBF itself or the SCFAs derived from GBF affect mast cells remains unclear. Further investigation is needed.

\section{References}

1. Stephen BH, Samuel M and David BS: The pharmacology of anti-inflammatory drugs in inflammatory bowel disease. In: Inflammatory Bowel Disease. 4th ed., Joseph BK and Roy GS (eds). Williams and Wilkins, Baltimore, pp643-694, 1995.

2. Griffiths AM, Ohlsson A, Sherman PM and Sutherland LR: Meta-analysis of enteral nutrition as a primary treatment of active Crohn's disease. Gastroenterology 108: 1056-1067, 1995.

3. Hond ED, Hiele M, Evenepoel P, Peeters M, Ghoos Y and Rutgeerts P: In vivo butyrate metabolism and colonic permeability in extensive colitis. Gastroenterology 115: 584-590, 1998.

4. Breuer RI, Buto SK, Christ ML, Bean J, Vernia P, Paoluzi P, Dipaolo MC and Caprilli R: Rectal irrigation with short chain fatty acids for distal ulcerative colitis preliminary report. Dig Dis Sci 36: 185-187, 1991.

5. Campieri M, Lanfranchi GA, Brignola C, Bazzocchi G, Gionchetti P, Minguzzi MR, Cappello IP, Corbelli C and Boschi S: Retrograde spread of 5-aminosalicylic acid enemas in patients with active ulcerative colitis. Dis Colon Rectum 29: 108-110, 1986.
6. Araki Y, Fujiyama Y, Andoh A, Koyama S, Kanauchi O and Bamba T: The dietary combination of germinated barley foodstuff plus Clostridium butyricum suppresses the dextran sulfate sodium-induced experimental colitis in rats. Scand J Gastroenterol 35: 1060-1067, 2000.

7. Kanauchi O, Mitsuyama K, Homma T, Takahama K, Fujiyama Y, Andoh A, Araki Y, Suga T, Hibi T, Naganuma M, Asakura H, Nakano H, Shimoyama T, Hida N, Haruma K, Koga H, Sata M, Tomiyasu N, Toyonaga A, Fukuda M, Kojima A and Bamba T: Treatment of ulcerative colitis patients by long-term administration of germinated barley foodstuff: Multi-center open trial. Int J Mol Med 12: 701-704, 2003.

8. Hanai H, Kanauchi O, Mitsuyama K, Andoh A, Takeuchi K, Takayuki I, Araki Y, Fujiyama Y, Toyonaga A, Sata M, Kojima A, Fukuda $\mathrm{M}$ and Bamba T: Germinated barley foodstuff prolongs remission in patients with ulcerative colitis. Int J Mol Med 13: 643-647, 2004.

9. Araki Y, Andoh A, Fujiyama Y, Kanauchi O, Takenaka K, Higuchi A and Bamba T: Germinated barley foodstuff exhibits different adsorption properties for hydrophilic versus hydrophobic bile acids. Digestion 64: 248-254, 2001.

10. Kanauchi O, Mitsuyama K, Araki Y and Andoh A: Modification of intestinal flora in the treatment of inflammatory bowel disease. Curr Pharm Des 9: 333-346, 2003.

11. Elson CO, Sartor RB, Tennyson GS and Riddell RH: Experimental models of inflammatory bowel disease. Gastroenterology 109: 1344-1367, 1995.

12. Kanauchi $\mathrm{O}$ and Agata K: Protein, and dietary fiber-rich new foodstuff from brewer's spent grain increased excretion of feces and jejunum mucosal protein content in rats. Biosci Biotechnol Biochem 61: 29-33, 1997.

13. Kanauchi O, Nakamura T, Agata K and Fushiki T: Preventive effect of germinated barley foodstuff on diarrhea induced by water-soluble dietary fiber in rats. Biosci Biotechnol Biochem 61: 449-454, 1997.

14. Araki Y, Andoh A, Fujiyama Y and Bamba T: Development of dextran sulphate sodium-induced experimental colitis is suppressed in genetically mast cell-deficient $W s / W s$ rats. Clin Exp Immunol 119: 264-269, 2000.

15. Oda T: Role of mast cells in dextran sulfate sodium-induced experimental colitis in rats. J Kyoto Pref Univ Med 104: 1069-1082, 1995.

16. Bene L, Sapi Z, Bajtai A, Buzas E, Szentmihalyi A, Arato A, Tulassay $Z$ and Falus A: Partial protection against dextran sodium sulphate induced colitis in histamine-deficient, histidine decarboxylase knockout mice. J Pediatr Gastroenterol Nutr 39: 171-176, 2004.

17. Okayama M, Tsubouchi R, Kato S and Takeuchi K: Protective effect of lafutidine, a novel histamine $\mathrm{H} 2$-receptor antagonist, on dextran sulfate sodium-induced colonic inflammation through capsaicin-sensitive afferent neurons in rats. Dig Dis Sci 49: 1696-1704, 2004.

18. Araki Y, Andoh A, Koyama S, Fujiyama Y, Kanauchi O and Bamba T: Effects of germinated barley foodstuff on microflora and short chain fatty acid production in dextran sulfate sodium-induced colitis in rats. Biosci Biotechnol Biochem 64: 1794-1800, 2000.

19. Kanauchi O, Agata K and Fushiki T: Mechanism for the increased defecation and jejunum mucosal protein content in rats by feeding germinated barley foodstuff. Biosci Biotech Biochem 61: 443-448, 1997.

20. Kanauchi O, Nakamura T, Agata K, Mitsuyama K and Iwanaga T: Effects of germinated barley foodstuff on dextran sulfate sodium-induced colitis in rats. J Gastroenterol 33: 179-188, 1998.

21. Roediger WEW: Utilization of nutrients by isolated epithelial cells of the rat colon. Gastroenterology 83: 424-429, 1982.

22. Kripke SA, Fox AD, Berman JM, Settle RG and Rombeau JL: Stimulation of intestinal mucosal growth and intracolonic infusion of short chain fatty acids. JPEN J Parenter Enteral Nutr 13: 109-116, 1989.

23. Andoh A, Fujiyama Y, Hata K, Araki Y, Takaya H, Shimada M and Bamba T: Counter-regulatory effect of sodium butyrate on tumour necrosis factor-alpha (TNF- $\alpha$ )-induced complement $\mathrm{C} 3$ and factor B biosynthesis in human intestinal epithelial cells. Clin Exp Immunol 118: 23-29, 1999.

24. Matsuhisa T, Mori Y and Tamura H: Sodium butyrate-induced adhesion in mastocytoma cells. Biol Cell 50: 1-7, 1984.

25. Koshihara Y, Kawamura M, Senshu T and Murota S: Effect of sodium n-butyrate on induction of prostaglandin synthase activity in cloned mastocytoma P-815 2-E-6 cells. Biochem J 194: 111-117, 1981. 